JOURNAL DE PHYSIQUE IV

Colloque C2, supplément au Journal de Physique III, Volume 5, février 1995

\title{
Spin Glass Model for the Study of Avalanches in Martensitic Transformations
}

\author{
E. Vives and A. Planes \\ Departament ECM, Facultat de Física, Universitat de Barcelona, Diagonal 647, 08028 Barcelona, \\ Catalonia, Spain
}

\begin{abstract}
We present a simplified model which reproduces the evolution through avalanches found in athermal martensitic transformations. The model is based on the Random Bond Ising model driven with an external field which mimics the effect of either the external stress or temperature. We have obtained power law distributions for the avalanche sizes in qualitative agreement with very recent experiments on martensitic transformations in $\mathrm{Cu}$ based alloys.
\end{abstract}

\section{INTRODUCTION}

Many metals and alloys exhibit Martensitic Tansformations (M.T.) between a B.C.C. phase and a compact phase. The transition is first order and usually can be induced either by decreasing the temperature or by applying an external stress. In this paper we focus in the systems exhibiting M.T. with thermoelastic behaviour [1]: contrarily to what happens in normal first order phase transitions the phase change is not abrupt but advances smoothly (in a macroscopic scale) when the temperature or external stress is changed displaying a very characteristic skew hysteresis cycle. This is due to the fact that the fraction of transformed material creates internal stresses which prevent the advance of the transformation in other parts of the system. Only when the temperature (or external stress) is changed enough, the free energy gain compensates the elastic energy and the transformation proceeds. Such behaviour is found, for instance, in a number of $\mathrm{Cu}$ based alloys like $\mathrm{Cu}-\mathrm{Zn}-\mathrm{Al}$ and $\mathrm{Cu}-\mathrm{Al}-\mathrm{Be}$.

Differential calorimetry [2] and acoustic emission measurements [3] which allow a careful study of the hysteresis cycle reveal that in a smaller scale the evolution proceeds through fast jumps joining local metastable states. There is little dependence on the kinetics (cooling-heating rate) of the driving parameter (temperature or stress), provided that the change is slow enough compared with the time scale for these jumps to occur. We call avalanches such jumps associated to the transformation of one or more domains in the system. In a recent study it has been pointed out that after a long number of cycles driven by temperature, the system sets into a situation with power law distribution of avalanche sizes [3]. Also, studies of the stress induced transition (between BCC and martensite or between martensite variants) have revealed the existence of avalanche behaviour. A similar behaviour has been found in magnetic materials driven with an external field (Barkhaussen effect) [4]. When the field is slowly reversed the ferromagnetic domains are flipped by avalanches distributed according to a power law. Three important factors seem to play a role for this behaviour 

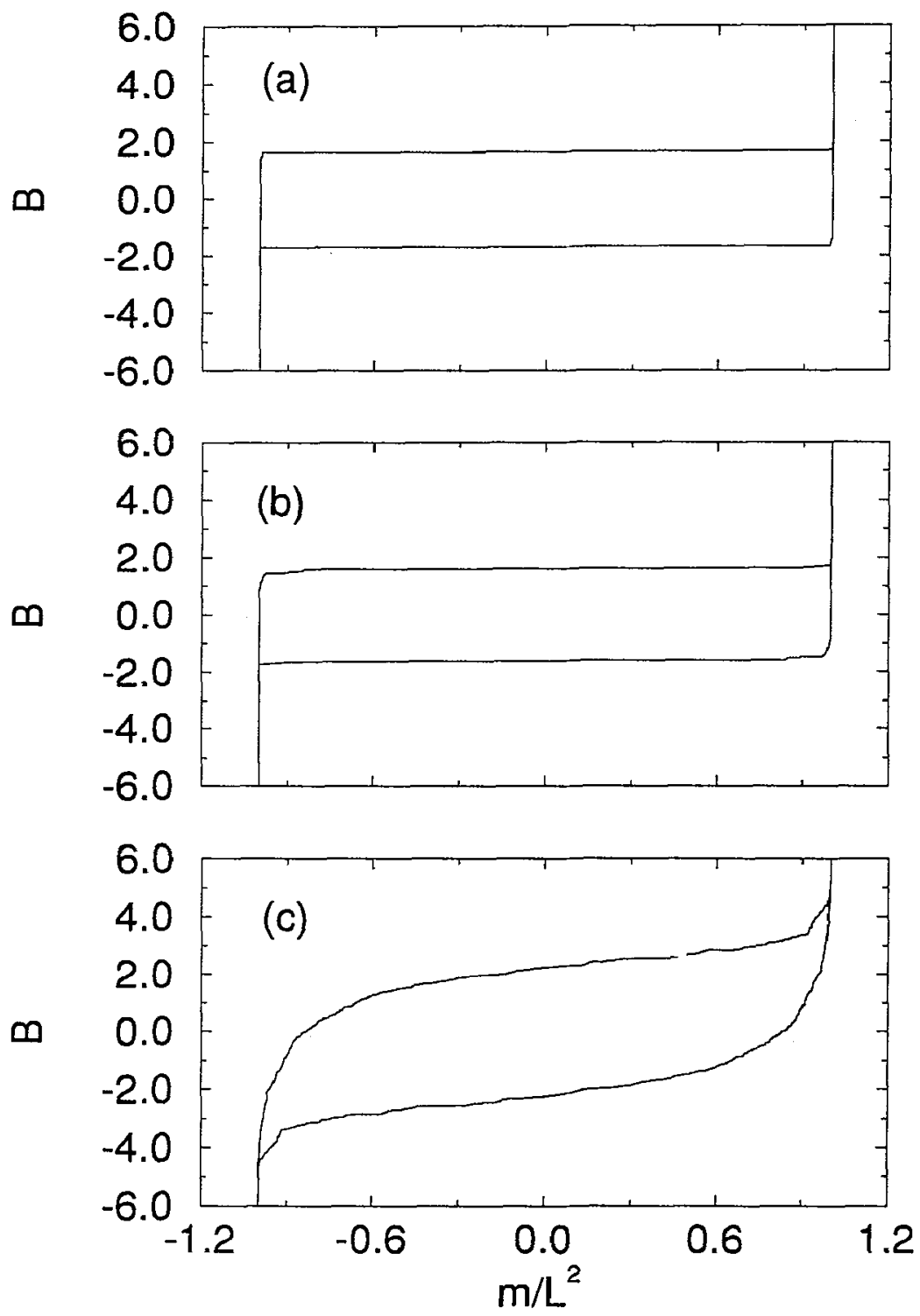

Figure 1: Examples of hysteresis cycles for different values of $\sigma:$ (a) $\sigma=0.45$, (b) $\sigma=0.55$, (c) $\sigma=1.5$. 
to appear. First the existence of quenched disorder (dislocations, vacancies, etc..) in the system which introduces nucleation centers for the new phase to appear and pinning centers stopping the advance of the interfaces. Second the existence of high energy barriers between the metastable states through which the system is advancing and finally the fact that thermal fluctuations do not play any important role. It is, however, not clear whether the role of long range interactions in the case of M.T. is important for this behaviour to appear or not. It can well be that its effect is just to introduce some correlations in the quenched disorder.

The aim of this paper is to present a very simple model [5] that can reproduce this behaviour and explain the power law distribution of the avalanches. The model is inspired in the Random Field Ising model introduced recently by Sethna et al. for the magnetic case [6]. The specific details of the model are described in section 2 . In section 3 we discuss the obtained hysteresis cycles and the distribution of avalanches. Finally in section 4 we present the main conclusions of the paper

\section{MODEL}

We consider the system divided into domains which form a regular lattice. For simplicity we restrict to an ideally shear induced transition between two oposite martensite variants. On each lattice site we define a spin variable $S_{i}$ that can take values 1 (one variant) or -1 (oposite variant). The proposed hamiltonian is the Random Bond Ising model with an external field which can be written as:

$$
H=\sum_{i, j} J_{i j} S_{i} S_{j}-B \sum_{i} S_{i},
$$

where the first sum extends over all nearest-neighbour pairs, the $J_{i j}$ are quenched interaction constants with gaussian random values with mean -1 and variable standard deviation $\sigma$. A pure ferromagnetic bond interaction $J=-1$ stabilizes neighbouring domains in the same variant. The disorder takes into account the existence of pinning or nucleation centers which locally favour or disfavour the ferromagnetic tendency. For large $\sigma$ an important fraction of the bonds will even change the sign and favour the stabilization of a domain wall between different variants.

The field $B$ reproduces the effect of an external shear driving the phase transition between both variants. For very large and positive $B$, one variant $(+1)$ is stabilized while for very negative values of $B$ the other variant $(-1)$ is the most stable one.

The simulations of this model start from the phase +1 for a very large external field $B$. The field $B$ is then decreased towards negative values until the flip of a spin to the new -1 variant represents a decrease in the total energy of the system. When such spin is flipped, it may unstabilize the neigbouring spins which are then sinchronously (and with the external field kept constant) reversed. These may unstabilize some more spins (avalanche effect) and so on until a new stable situation is reached. Then the field $B$ is decreased until a new avalanche starts. The total number of spins involved in one avalanche will be called size of the avalanche.

The model retains a lot of good properties from the M.T. experiments. Nevertheless some criticisms and improvements can be stated. First the description of the domain structrue with a $2 \mathrm{~d}$ lattice is far from reality. A generalization to higher dimensions is straight forward. An still better improvement with a random structure of domains with more long range interactions can in fact be studied since it corresponds to a mean field version of the model we are proposing. In fact the Random Bond Ising model with zero mean interaction has been used for the study of spin-glasses [7] (Edwards-Anderson model) and solved in Mean Field approximation some years ago (SherringtonKirkpatrik model). The generalization of the Sherrington-Kirkpatrik solution to our case with a mean ferromagnetic interaction might be possible in the near future. The model can also be extended to the temperature induced martensitic transformation from the $\mathrm{BCC}$ to the martensitic phase. This martensitic phase is known to exhibit a larger degeneracy (a big number of variants) compared to the low degeneracy of the high temperature BCC phase. This can make a lot of differences between 

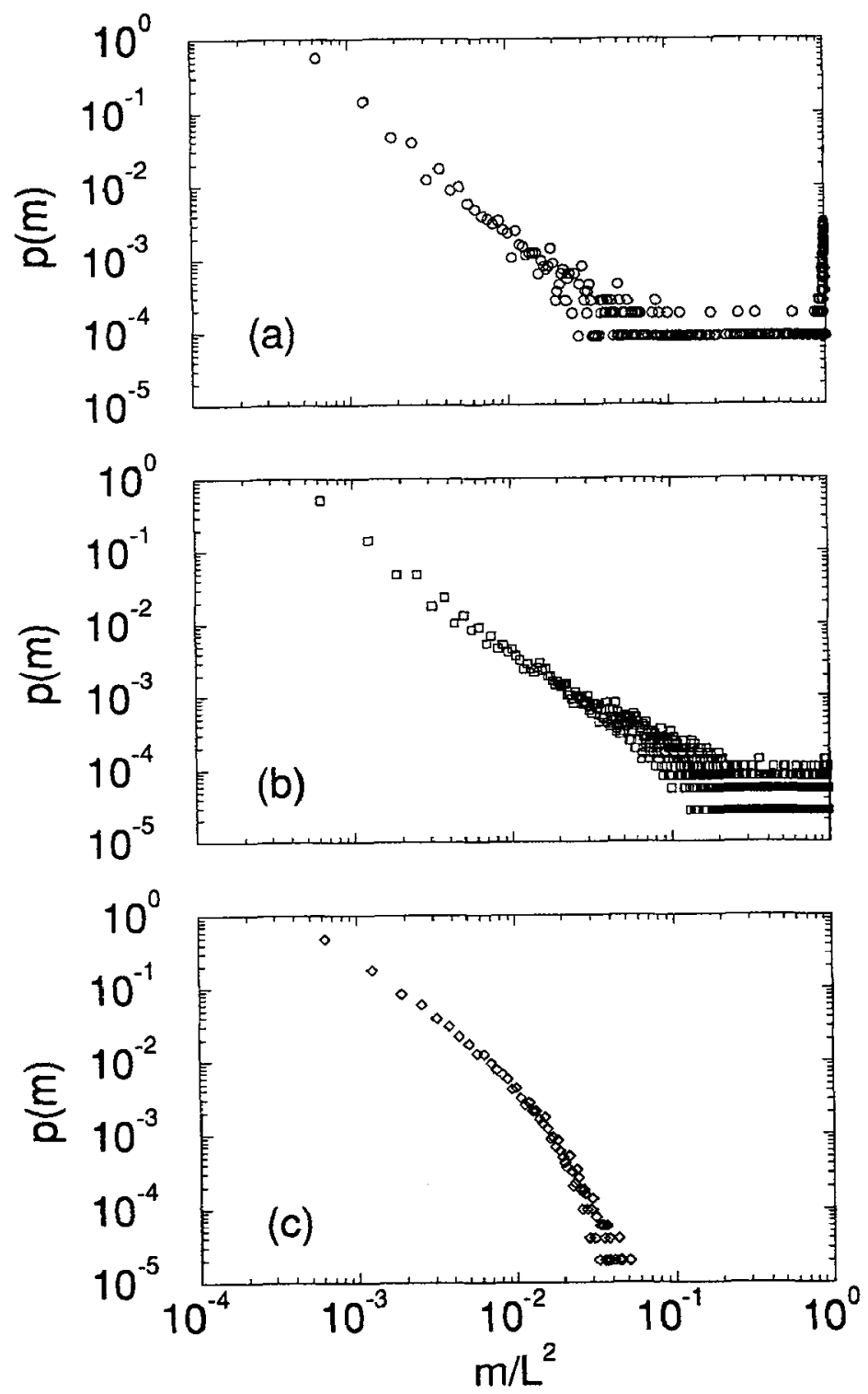

Figure 2: Distribution of avalanche sizes for the same values of $\sigma$ of the previous figure. 
the forward and reverse branches of the hysteresis cycle which cannot be reproduced by our symmetrical model. An improvement of the model will be to substitute the \pm 1 variables by spins with more states, for instance $S_{i}=-1,0,1$, with 0 representing the high temperature BCC phase and \pm 1 representing two variants of the low temperature phase. Such a model is also presented in these proceedings [8].

The simulations have been done on lattices with different side sizes $(L=10,15,20,30,40,60,80,100)$. For each size different values of $\sigma$ have been analyzed and for each $\sigma$ a large number of runs $(\sim 500)$ starting with different realizations of the random bonds have been studied.

\section{RESULTS}

In order to study the evolution of the system we have monitored the magnetization as a function of the external field when cycling. The cycles always show an inversion center symmetry due to the symmetry of the hamiltonian. Figure 1 shows, as an example, three hysteresis cycles corresponding to a system of size $L=40$ and $\sigma=0.45(\mathrm{a}), \sigma=0.55(\mathrm{~b})$ and $\sigma=1.5(\mathrm{c})$. For low values of $\sigma$, the hysteresis loop shows very few tiny jumps and a very big macroscopic avalanche involving almost all the spins in the system. For very large values $\sigma>0.7$ no macroscopic avalanches can be distinguished and the hysteresis loop looks skew and smooth. Figure 2 shows a log-log plot of the distribution of avalanche sizes (magnetization change) corresponding to the same three cases as in fig. 1. Such distributions are obtained from the simulation of 500 independent runs. For small values of $\sigma$ a delta-like peak can be distinguished for a large value of $m$ corresponding to the large avalanche. Also some small avalanches are present which are responsible for the rounding off of the hysteresis cycles before and after the big jump. For large values of $\sigma$ the histogram shows that only small avalanches are present in the system. In the intermediate region, there is a value of $\sigma$ (close to $\sigma=0.55$ ) for which the distribution of avalanches shows a power-law behaviour indicating the absence of intrinsic scales in the system. This behaviour is indicative of the existence of criticality at this point. Standard finite size scaling analysis allows to obtain the value of $\sigma$ at which the critical distribution appears for an infinite system $\left(\sigma_{c}=0.45\right)$, and the slope of such critical distribution of the avalanche sizes $\tau=1.45$.

Other interesting features that the model reproduces are the behaviour of the partial loops in the hysteresis cycle. Figure 3 shows such an example in which the interesting Return Point Memory property [9] is not satisfied: after the partial cycling, the evolution of the system is not the same as for the case without partial cycle.

\section{CONCLUSIONS}

From the above results one can conclude that the model describes satisfactorily the dependence of the hysteresis cycle with the amount of disorder in the system. Indeed, experimentally, it is found that for fast quenched samples (with a lot of disorder, large $\sigma$ ) the hysteresis cycle looks smooth as in figure 1c. For slowly quenched samples (less disorder, small $\sigma$ ) the hysteresis loop is sharper. In between there exists a situation which would be accessed after cycling enough, caracterized by a final attractor for the hysteresis cycle with critical behaviour (power law distribution of the avalanche sizes). About the return point memory property, our model (RBIM) does not show it while it is known that it is exhibited by the similar model (Random Field Ising model) proposed by Sethna et al. [6]. The reason for this behaviour may sit on the fact that in our case a fraction of the bonds is of antiferromagnetic type (stabilizes two neigbouring different variants). This may induce the occurence of some reverse transformations while driving the external field monotonously which destroys the partial order of the states necessary for the Return Point Memory property to appear. Such reverse transformations have been found experimentally in $\mathrm{Cu}-\mathrm{Zn}-\mathrm{Al}$ [10].

We acknowledge J.Sethna for useful discussions and finantial support from CICyT (Spain) under project number MAT92-884. 


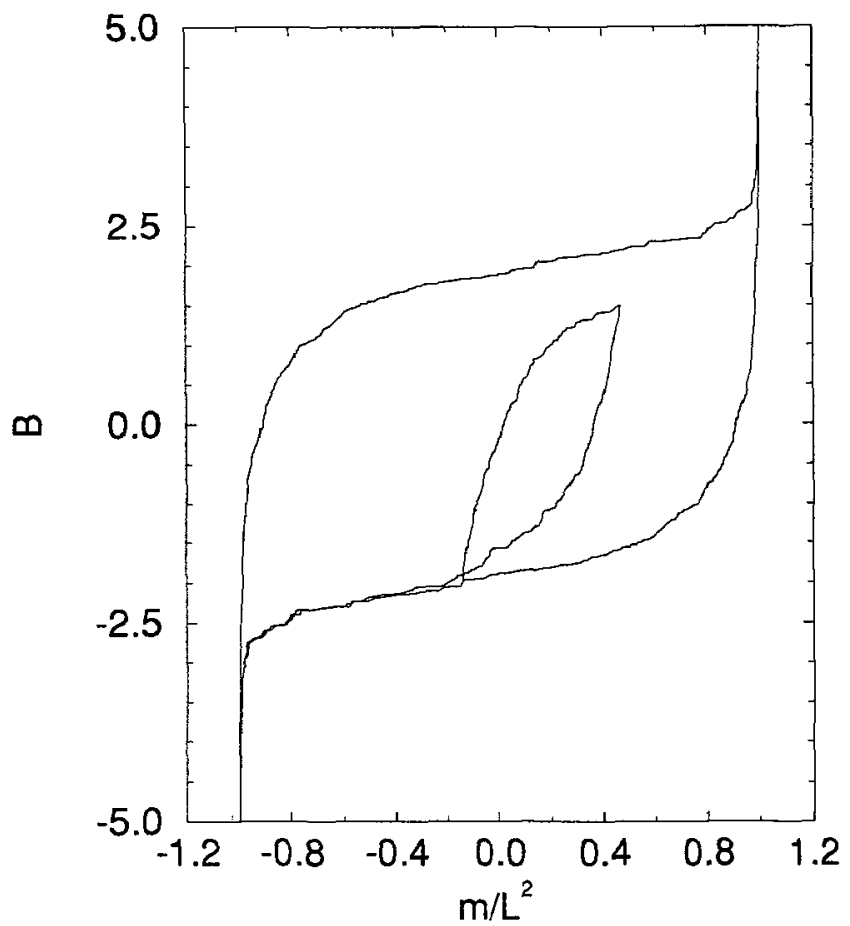

Figure 3: Example of hysteresis cycle showing no return point memory property

\section{References}

[1] Delaey L., Krishnan H., Tas H. and Warlimont H., J. Mater Sci. 9 (1974) 1521-1550.

[2] Planes A., Macqueron J.L., Morin M. and Guénin G., Phys. Stat. Sol. (a) 66 (1981) 717-724.

[3] Vives E., Ortín J., Mañosa L., Ràfols I., Pérez-Magrané R. and Planes A., Phys. Rev. Lett. 72 (1994)1694-1697.

[4] Cote P.J. and Meisel L.V., Phys. Rev. Lett. 67 (1991) 1334-1337.

[5] Vives E. and Planes A., Phys. Rev. B 50 (1994) in press.

[6] Sethna J.P., Dahmen K., Kartha S., Krumhansl J.A., Roberts B.W. and Shore J.D., Phys. Rev. Lett. 70 (1993) 3347-3350.

[7] Binder K. and Young A.P., Rev. Mod. Phys. 58 (1986) 801-976.

[8] Goicoechea J. and Ortín J., "A Random Field 3-state spin model to simulate hysteresis and avalanches in martensitic transformations." in these proceedings.

[9] Ortín J., J. Appl. Phys. 71 (1992) 1456-1462. and references therein.

[10] J. Ortín showed us a sequence of photographs where this effect could be easily observed when the temperature was monotonously decreased. 